\title{
Blood-brain barrier derangement after electrical brain stimulation
}

\author{
Yumiko Watanabe ${ }^{1}$, N.A.R. Nik-Mohd-Afizan ${ }^{1,2}$, Ichiro Takashima ${ }^{1 *}$
}

'Human Informatics Research Institute, National Institute of Advanced Industrial Science and Technology, 1-1-1 Umezono, Tsukuba 305-8568, Japan

${ }^{2}$ Faculty of Biotechnology and Biomolecular Sciences, Universiti Putra Malaysia, 43400 Serdang, Selangor, Malaysia

\section{Article Info}

\section{Article Notes}

Received: November 01, 2017

Accepted: November 20, 2017

\section{*Correspondence:}

Dr. Ichiro Takashima

Human Informatics Research Institute, National Institute of

Advanced Industrial Science and Technology (AIST),

1-1-1 Umezono, Tsukuba, Ibaraki 305-8568, Japan

Tel: +81-29-861-5563, Fax: +81-29-861-5849, Email:

i.takashima@aist.go.jp

(c) 2017 Takashima I. This article is distributed under the terms of the Creative Commons Attribution 4.0 International License

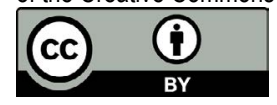

\section{Keywords}

Electric brain stimulation

Transcranial direct current stimulation (tDCS)

Blood brain barrier (BBB)

BBB permeability

Neurological disorders

Drug delivery

Therapeutic window

\section{ABSTRACT}

Noninvasive brain stimulation methods, including repetitive transcranial magnetic stimulation and transcranial direct current stimulation (tDCS), have received considerable attention in recent years for use in the study and treatment of neurological conditions. Of these methods, tDCS is considered particularly promising due to its ease of use and ability to confer polarity-dependent effects on brain excitability, making it an excellent option for clinical treatment of neurological and psychiatric diseases. While generally regarded as safe when following standard protocols, the effects of tDCS on cerebral blood vessels and blood-brain barrier (BBB) functions remain poorly understood. Here, we provide an overview of tDCS in the context of BBB function, summarize the current literature, and discuss implications for future research. To date, no alterations or damage to the BBB have been reported after weak tDCS stimulations in human subjects; however, some animal studies have reported alterations to BBB function following increased tDCS intensity, with inconsistencies in the effective tDCS polarity used to produce these BBB disruptions between studies. Further research will be necessary to evaluate the effects of tDCS on the BBB under various conditions. Finally, we discuss the potential of tDCS for enhancing drug delivery to the central nervous system, which may become possible as we refine our understanding of the effects of tDCS on BBB permeability.

\section{Background}

Noninvasive brain stimulation methods, including repetitive transcranial magnetic stimulation (rTMS) and transcranial direct current stimulation (tDCS), have received considerable attention in recent years ${ }^{1-3}$. These methods are considered a new alternative to standard pharmacotherapy, and have been used to treat a wide range of neurological and psychiatric diseases, including post-stroke disorders, depression, Alzheimer's disease, and Parkinson's disease ${ }^{4-9}$. In these treatments, stimulation with magnetic (rTMS) or direct electrical (tDCS) energy interacts with the cortical neurons, resulting in excitement or inhibition of neuronal activity in a specific brain region ${ }^{10-13}$. Among these two methods, rTMS offers better spatial and temporal resolution than $\mathrm{tDCS}^{14,15}$, but carries a greater risk of inducing seizures and requires a higher degree of both technical and clinical expertise ${ }^{16,17}$. In contrast, tDCS is less expensive than rTMS; a typical tDCS apparatus is batteryoperated, highly portable, and easy to use. In some clinical settings, the effects of tDCS appear to last longer than those of rTMS ${ }^{18,19}$. Furthermore, tDCS has been shown to confer polarity-dependent effects on brain excitability. Specifically, anodal stimulation facilitates the depolarization of neurons, while cathodal stimulation hyperpolarizes the resting membrane potential and reduces neuronal firing ${ }^{20-23}$. Taking these 
findings together, this ability to modulate the excitatory/ inhibitory balance of the brain in a convenient, easy-to-use system makes tDCS a promising approach for the treatment of neuropsychiatric symptoms.

\section{Safety}

The safety of tDCS has been well established in recent years ${ }^{24}$. To date, no severe adverse effects have been reported using standard tDCS protocols, although significant care should still be used when applying tDCS in clinical settings. During tDCS, mild tingling and itching sensations under the electrode are the most common side effects, with some patients complaining of a hot or burning sensation, and pain ${ }^{25-28}$. With high current amplitudes, redness and slight burning of the skin under the electrode are common ${ }^{29,30}$; other symptoms such as headache, fatigue, nausea, insomnia, and mood changes after tDCS have also been noted ${ }^{25}$. But thus far, seizure incidents have not been reported in tDCS studies. Overall, tDCS is considered safe when following established protocols ${ }^{24,31,32}$; however, the effects of tDCS on cerebral blood vessels and blood-brain barrier (BBB) functions remain poorly understood.

\section{Effects on BBB integrity}

The BBB is a highly selective interface that separates the brain from circulating blood, preventing many viruses, bacteria, and toxins from entering the central nervous system. Maintenance of the BBB is therefore of critical importance, both during and after tDCS therapy.

To date, only a small number of studies have investigated the safety of tDCS with respect to blood-brain barrier disturbance. Nitsche et al. applied weak tDCS stimulation to healthy human subjects and used MRI to test for the possibility of structural changes in brain tissue or alteration of the $\mathrm{BBB}^{33}$. In this study, $1 \mathrm{~mA}$ current was applied to the frontal cortex through saline-soaked sponge electrodes (0.03 $\mathrm{mA} / \mathrm{cm}^{2}$ ) for 9-13 min. Subsequent MRI scans performed 30 and 60 min after tDCS revealed no evidence of brain edema, structural changes in cerebral tissue, or alterations of the $\mathrm{BBB}^{33}$. However, given the weak stimulus conditions used in this study ( $1 \mathrm{~mA}$ for up to $13 \mathrm{~min})^{33}$, these results alone are insufficient to establish the safety of tDCS in the context of cerebral tissues or BBB integrity, as conventional tDCS protocols employ current intensities up to $4 \mathrm{~mA}$, with durations ranging up to $40 \mathrm{~min}^{24}$. The relationship between stimulus intensity and outcome is not clear yet, but a couple of studies suggested that stronger stimulation may result in greater benefits. Iyer et al. examined the effects of tDCS on cognitive function in healthy human subjects and found that verbal fluency significantly improved following stimulation at $2 \mathrm{~mA}$, compared with no significant effects after stimulation with $1 \mathrm{~mA}^{34}$. Similarly, $\mathrm{Yu}$ et al. observed significant improvements in spatial learning and memory under strong stimulus conditions $\left(>3.2 \mathrm{~mA} / \mathrm{cm}^{2}\right)$ using a rat model of Alzheimer's disease $\mathrm{e}^{35}$. Given these apparent intensity-dependent effects of tDCS treatment, it is important to determine the effect of tDCS on the BBB integrity when using relatively high current stimulations.

A recent study reported by Nik-Mohd-Afizan et al. investigated the effect of high current tDCS in rats, and found that anodal, but not cathodal, tDCS had negligible effects on BBB permeability ${ }^{36}$. Here, anodal stimulation was applied at a charge density of $260,820 \mathrm{C} / \mathrm{m}^{2}$, an intensity five times higher than the threshold estimate for cathodal tDCS-induced brain damage in rats ${ }^{37}$. BBB permeability was assessed by quantifying extravasation of Evans Blue dye (EBD) delivered via intravenous injection immediately follow tDCS treatment. Results from these experiments showed that cathodal stimulation produced significant extravasation of EBD, compared with minimal leakage in response to anodal stimulation, with anodal stimulation outcomes not significantly different from those in shamstimulated controls ${ }^{36}$. Further validation of these results was performed by immunohistochemical staining of brain tissues obtained after tDCS stimulation for claudin-5 (CLDN5), a key component sealing the paracellular space between adjacent endothelial cells ${ }^{38}$. These analyses revealed significantly decreases in CLDN5 levels following cathodal, but not anodal, stimulation, indicating that anodal stimulation did not affect tight junction permeability ${ }^{36}$. Taken together, these findings reveal polarity-specific effects of brain stimulation on the BBB permeability, and suggest that anodal tDCS may be used safely at increasing stimulus intensity.

Despite the strong evidence reported by Nik-MohdAfizan et al., a different study using a mouse ischemic stroke model reported that anodal tDCS exacerbated dysregulation of BBB during the acute phase of stroke ${ }^{39}$. In this study, Peruzzotti-Jametti et al. evaluated the ratio of endogenous immunoglobulin G (IgG) extravasation in the ipsilateral ischemic hemisphere, and found a significant increase in endogenous IgG leakage only in the anodal tDCS group ${ }^{39}$. They also analyzed the endothelial tight junction protein zona occludens-1 (ZO-1) and confirmed a significant decrease in its expression in the anodal tDCS condition. As ZO-1 is fundamental for maintaining the BBB after ischemic stroke ${ }^{40,41}$, these results suggest that the disruption of blood vessel tight junctions occurs in the case of anodal stimulation, but not cathodal stimulation ${ }^{39}$. To put these results in context, these findings were obtained using a charge density of $132,000 \mathrm{C} / \mathrm{m}^{2}$ for tDCS stimulation, which was about half of that used by Nik-Mohd-Afizan et $a .^{36}$.

Discrepancies in the polarity effects of high-intensity tDCS on BBB disruption mark an important distinction between these two studies. One possible explanation 
for this discrepancy could be that Nik-Mohd-Afizan et al. performed tDCS on normal healthy brains ${ }^{36}$, whereas Peruzzotti-Jametti et al. ${ }^{39}$ applied tDCS to the ischemic brain in the acute phase of stroke. These findings suggest that the safety of tDCS may differ between healthy and injured brains, with the severity of brain damage or post-acute/ chronic stages of brain injury affecting patient outcomes. Further animal studies will be necessary to resolve this issue.

\section{Potential for drug delivery}

While maintenance of the BBB is of crucial importance for long-term neuronal health, it represents a formidable obstacle to drug delivery, limiting the effectiveness of many drugs used to treat neurological disorders, such as brain tumors, Alzheimer's disease, and Parkinson's disease. Therefore, methods capable of temporarily improving the permeability of drugs across the BBB may hold considerable promise for the treatment of neurological disorders. Existing treatments, such as the administration of hyperosmotic agents (e.g., mannitol) or blood vessel dilators (e.g., bradykinin), have been shown to increase BBB permeability, providing an enhanced therapeutic window lasting several hours ${ }^{42,43}$. On top of that, direct targeting methods using lasers, focused ultrasound, or hyperthermia have also been evaluated as an alternative way to create a transient opening in the $\mathrm{BBB}^{44,45}$.

Considerable evidence indicates that electrical brain stimulation methods, such as tDCS affect a range of cerebrovascular functions ${ }^{46}$ including BBB integrity, as described above. Assuming that the correct stimulus parameters can be identified, tDCS may become a viable and feasible option for modulating drug delivery to the CNS. Recent work by Shin et al. provides an important proof of concept, having shown that brief exposure (20 min, $1 \mathrm{~mA}$ ) of the rat frontal cortex to tDCS was able temporarily to increase the permeability of cerebral microvessels to small and large molecules (molecular weight ranging from 376 to 70,000 ), with BBB permeability returning to baseline levels within 20 min post-treatment ${ }^{47}$. These results highlight the considerable potential of tDCS for drug delivery; however, more research will be needed to evaluate the overall efficacy of this procedure, and the safety of tDCS on the cortical vasculature.

\section{Future perspective}

In clinical MRI, gadolinium-based contrast agents have been used as a reliable marker of impaired $\mathrm{BBB}^{48}$. However, as an alternative approach, recent studies have reported several biomarkers specific to BBB disruption in serum of patients (e.g. astrocyte-derived protein S-100 $\beta^{49}$ and tight junction protein $\mathrm{CLDN}^{50}$ ). Assuming such proteins circulating in the blood mirror BBB integrity, accumulated evidence from animal studies may be useful in clinical trials. Therefore, future studies examining tDCS effects on BBB permeability in animal models of human diseases should combine measurement of serum levels of candidate clinical biomarkers.

\section{Conclusion}

Here, we have presented a brief summary focusing the effects of tDCS on BBB permeability. Although tDCS is a convenient technique capable of modulating brain functions, its effects on cerebrovascular and other neurological functions remain poorly understood. Furthermore, additional research will be necessary to assess both the efficacy of this procedure and the safety of tDCS on the cortical vasculature. Further advances in our understanding of tDCS and its effects on BBB function may unlock the potential of this method for improving drug delivery to the CNS. Given the clear benefits of electrical brain stimulation methods for the treatment of neurological symptoms, a combination of drug therapy and electrical brain stimulation may offer considerable promise for the treatment of neurological disorders in future.

\section{Acknowledgement}

This work was supported by grants from the Ministry of Education, Culture, Sports, Science and Technology of Japan [15K12780 and 16K04443].

\section{Conflict of Interest}

The authors declare no conflict of interest.

\section{References}

1. Wagner T, Valero-Cabre A, Pascual-Leone A. Noninvasive human brain stimulation. Annu Rev Biomed Eng. 2007; 9: 527-565.

2. Brunoni AR, Nitsche MA, Bolognini N, et al. Clinical research with transcranial direct current stimulation (tDCS): challenges and future directions. Brain Stimul. 2012; 5(3): 175-195.

3. Jackson MP, Rahman A, Lafon B, et al. Animal models of transcranial direct current stimulation: Methods and mechanisms. Clin Neurophysiol. 2016; 127(11): 3425-3454.

4. Feng WW, Bowden MG, Kautz S. Review of transcranial direct current stimulation in poststroke recovery. Top Stroke Rehabil. 2013; 20(1): 68-77.

5. Kuo MF, Paulus W, Nitsche MA. Therapeutic effects of non-invasive brain stimulation with direct currents (tDCS) in neuropsychiatric diseases. Neuroimage. 2014; 85(Pt3): 948-960.

6. Broeder S, Nackaerts E, Heremans E, et al. Transcranial direct current stimulation in Parkinson's disease: Neurophysiological mechanisms and behavioral effects. Neurosci Biobehav Rev. 2015; 57: 105-117.

7. Kang N, Summers JJ, Cauraugh JH. Non-Invasive Brain Stimulation Improves Paretic Limb Force Production: A Systematic Review and Meta-Analysis. Brain Stimul. 2016; 9(5): 662-670.

8. Hsu WY, Ku Y, Zanto TP, et al. Effects of noninvasive brain stimulation on cognitive function in healthy aging and Alzheimer's disease: a systematic review and meta-analysis. Neurobiol Aging. 2015; 36(8): 2348-2359.

9. Dinkelbach L, Brambilla M, Manenti R, et al. Non-invasive brain 
stimulation in Parkinson's disease: Exploiting crossroads of cognition and mood. Neurosci Biobehav Rev. 2017; 75: 407-418.

10. Hoogendam JM, Ramakers GM, Di Lazzaro V. Physiology of repetitive transcranial magnetic stimulation of the human brain. Brain Stimul. 2010; 3(2): 95-118.

11. Lenz M, Vlachos A. Releasing the Cortical Brake by Non-Invasive Electromagnetic Stimulation? rTMS Induces LTD of GABAergic Neurotransmission. Front Neural Circuits. 2016; 10: 96.

12. Nitsche MA, Paulus W. Excitability changes induced in the human motor cortex by weak transcranial direct current stimulation. Physiol. 2000; 527(Pt3): 633-639.

13. Nitsche MA, Nitsche MS, Klein CC, et al. Level of action of cathodal DC polarisation induced inhibition of the human motor cortex. Clin Neurophysiol. 2003; 114(4): 600-604.

14. Gandiga PC, Hummel FC, Cohen LG. Transcranial DC stimulation (tDCS): a tool for double-blind sham-controlled clinical studies in brain stimulation. Clin Neurophysiol. 2006; 117(4): 845-850.

15. Zimerman M, Hummel FC. Non-Invasive Brain Stimulation: Enhancing Motor and Cognitive Functions In Healthy Old Subjects. Front Aging Neurosci. 2010; 2: 149.

16. Loo CK, McFarquhar TF, Mitchell PB. A review of the safety of repetitive transcranial magnetic stimulation as a clinical treatment for depression. Int J Neuropsychopharmacol. 2008; 11(1): 131-147.

17. Dobek CE, Blumberger DM, Downar J, et al. Risk of seizures in transcranial magnetic stimulation: a clinical review to inform consent process focused on bupropion. Neuropsychiatr Dis Treat. 2015; 11:2975-2987.

18. Fregni F, Boggio PS, Lima MC, et al. A sham-controlled, phase II trial of transcranial direct current stimulation for the treatment of centra pain in traumatic spinal cord injury. Pain. 2006; 122(1-2): 197-209.

19. Vestito L, Rosellini S, Mantero M, et al. Long-Term Effects of Transcranial Direct-Current Stimulation in Chronic Post-Stroke Aphasia: A Pilot Study. Front Hum Neurosci. 2014; 8: 785.

20. Medeiros LF, de Souza IC, Vidor LP, et al. Neurobiological effects of transcranial direct current stimulation: A review. Front Psychiatry. 2012; 3: 110 .

21. Purpura DP, McMurty JG. Intracellular activities and evoked potential changes during polarization of motor cortex. J Neurophysiol, 1965; 28: $166-185$

22. Chan CY, Hounsgaard J, Nicholson C. Effects of electric fields on transmembrane potential and excitability of turtle cerebellar Purkinje cells in vitro. J Physiol. 1988; 402: 751-771.

23. Bindman LJ, Lippold OC, Redfearn JW. The action of brief polarizing currents on the cerebral cortex of the rat (1) during current flow and (2) in the production of long-lasting after-effects. J Physiol. 1964; 172(3): 369-382.

24. Bikson M, Grossman P, Thomas C, et al. Safety of Transcranial Direct Current Stimulation: Evidence Based Update 2016. Brain Stimul. 2016; 9(5): 641-661.

25. Poreisz C, Boros K, Antal A, et al. Safety aspects of transcranial direct current stimulation concerning healthy subjects and patients. Brain Res Bull. 2007; 72(4-6):208-214.

26. Furubayashi T, Terao Y, Arai N, et al. Short and long duration transcranial direct current stimulation (tDCS) over the human hand motor area. Exp Brain Res. 2008; 185(2): 279-286.

27. Brunoni AR, Amadera J, Berbel B, et al. A systematic review on reporting and assessment of adverse effects associated with transcranial direct current stimulation. Int J Neuropsychopharmacol. 2011; 14(8): 1133-1145.
28. Matsumoto $\mathrm{H}$, Ugawa Y. Adverse events of tDCS and tACS: A review. Clin Neuropysiol Pract. 2017; 2: 19-25.

29. Palm U, Keeser D, Schiller C, et al. Skin lesions after treatment with transcranial direct current stimulation (tDCS). Brain Stim. 2008; 1(4): 386-387.

30. Frank E, Wilfurth S, Landgrebe $M$, et al. Anodal skin lesions after treatment with transcranial direct current stimulation. Brain Stim. 2010: 3(1): 58-59.

31. Antal A, Alekseichuk I, Bikson M, et al. Low intensity transcranial electric stimulation: Safety, ethical, legal regulatory and application guidelines. Clin Neurophysiol. 2017; 128(9):1774-1809.

32. Zhao H, Qiao L, Fan D, et al. Modulation of Brain Activity with Noninvasive Transcranial Direct Current Stimulation (tDCS): Clinical Applications and Safety Concerns. Front Psychol. 2017; 8:685.

33. Nitsche MA, Niehaus L, Hoffmann KT, et al. MRI study of human brain exposed to weak direct current stimulation of the frontal cortex. Clin Neurophysiol. 2004; 115(10): 2419-2423.

34. Iyer MB, Mattu U, Grafman J, et al. Safety and cognitive effect of frontal DC brain polarization in healthy individuals. Neurology. 2005; 64(5): 872-875.

35. Yu X, Li Y, Wen H, et al. Intensity-dependent effects of repetitive anodal transcranial direct current stimulation on learning and memory in a rat model of Alzheimer's disease. Neurobiol Learn Mem. 2015; 123: 168-178.

36. Nik-Mohd-Afizan NAR, Watanabe Y, Takashima I. Safety of the anodal direct current stimulation on the permeability of the rat blood-brain barrier. Curr Neurobiol. 2017; 8(2): 34-39.

37. Liebetanz D, Koch R, Mayenfels S, et al. Safety limits of cathodal transcranial direct current stimulation in rats. Clin Neurophysiol. 2009; 120(6): 1161-1167.

38. Wallez Y, Huber P. Endothelial adherens and tight junctions in vascular homeostasis, inflammation and angiogenesis. Biochim Biophys Acta. 2008; 1778(3): 794-809.

39. Peruzzotti-Jametti L, Cambiaghi M, Bacigaluppi M, et al. Safety and efficacy of transcranial direct current stimulation in acute experimental ischemic stroke. Stroke. 2013; 44(11): 3166-3174.

40. Yilmaz G, Granger DN. Leukocyte recruitment and ischemic brain injury. Neuromolecular Med. 2010; 12(2): 193-204.

41. Abbott NJ, Patabendige AA, Dolman DE, et al. Structure and function of the blood-brain barrier. Neurobiol Dis. 2010; 37(1): 13-25.

42. Rapoport SI. Osmotic opening of the blood-brain barrier: principles, mechanism, and therapeutic applications. Cell Mol Neurobiol. 2010; 20(2): 217-230.

43. Liu LB, Xue YX, Liu YH. Bradykinin increases the permeability of the blood-tumor barrier by the caveolae-mediated transcellular pathway. J Neurooncol. 2010; 99(2): 187-194.

44. Choi M, Ku T, Chong K, et al. Minimally invasive molecular delivery into the brain using optical modulation of vascular permeability. Proc Natl Acad Sci U S A. 2011; 108(22): 9256-9261.

45. Carpentier A, Canney M, Vignot A, Clinical trial of blood-brain barrier disruption by pulsed ultrasound. Sci Transl Med. 2016; 8(343): 343re2.

46. Pulgar VM. Direct electrical stimulation to increase cerebrovascular function. Front Syst Neurosci. 2015; 9: 54

47. Shin DW, Khadka N, Fan J, et al. Transcranial direct current stimulation transiently increases the blood-brain barrier solute permeability in vivo. Proc SPIE Medical Imaging. 2016; 97881X.

48. Montagne A, Toga AW, Zlokovic BV. Blood-brain Barrier Permeability and Gadolinium: Benefits and Potential Pitfalls in Research. AMA Neurol. 2016; 73(1): 13-14. 
49. Kapural M, Krizanac-Bengez Lj, Barnett G et al. Serum S-100ß as a possible marker of blood-brain barrier disruption. Brain Research. 2002; 940(1-2): 102-104
50. Kazmierski R, Michalak S, Wencel-Warot A, et al. Serum tight-junction proteins predict hemorrhagic transformation in ischemic stroke patients. Neurology. 2012; 79(16): 1677-1685. 